\title{
THE GLOBULAR CLUSTER SYSTEM OF THE LOW-LUMINOSITY ELLIPTICAL GALAXY NGC 1427
}

\author{
JUAN C. Forte \\ Facultad de Ciencias Astronómicas y Geofísicas, Universidad Nacional de La Plata, Paseo del Bosque s/n, 1900 La Plata, Argentina, and CONICET; \\ forte@fcaglp.unlp.edu.ar \\ DOUG GEISLER \\ Departamento de Física, Grupo de Astronomía, Universidad de Concepción, Casilla 160-C, Concepción, Chile; doug@kukita.cfm.udec.cl \\ Pablo G. Ostrov \\ Facultad de Ciencias Astronómicas y Geofísicas, Universidad Nacional de La Plata, Paseo del Bosque s/n, 1900 La Plata, Argentina; ostrov@fcaglp.edu.ar \\ Andrés E. Piatti \\ Observatorio Astronómico, Universidad Nacional de Córdoba, Laprida 854, 5000 Córdoba, Argentina; andres@mail.oac.uncor.edu \\ AND \\ WOLFGANG GIEREN \\ Departamento de Física, Grupo de Astronomía, Universidad de Concepción, Casilla 160-C, Concepción, Chile; wgieren@coma.cfm.udec.cl \\ Received 2000 October 13; accepted 2000 December 20
}

\begin{abstract}
Washington photometry is presented for a large number of globular cluster candidates associated with the low-luminosity elliptical galaxy NGC 1427 in the Fornax cluster. The survey is mostly complete to $T_{1}=23.5(V \approx 24.0)$ and includes an areal coverage of about $216 \mathrm{arcmin}^{2}$, centered near the galaxy. Most previous studies have failed to detect any evidence of multiple globular cluster populations in this low-luminosity elliptical, in sharp contrast to the bimodal globular cluster systems commonly found in giant ellipticals. The lack of multimodal cluster populations has been used as the basis for suggesting that the formation mechanisms for low-luminosity and giant ellipticals are significantly different. Our metallicity-sensitive $C-T_{1}$ photometry (the first such study of a low-luminosity elliptical) reveals a definite bimodal cluster population. The red globular cluster population appears strongly centrally concentrated and practically disappears beyond a galactocentric radius of $120^{\prime \prime}$. The mean color of these clusters is similar to that of the inner galaxy halo. Blue globulars, on the other hand, exhibit a shallower spatial distribution. These clusters share a small negative $C-T_{1}$ color gradient with the galaxy halo, although they are, on average, some $0.3 \mathrm{mag}$ bluer at all galactocentric radii. The overall mean cluster system metallicity is $-0.9 \pm 0.2$. The specific globular cluster frequency $S_{N}$ is $4.5 \pm 0.8$, if a distance modulus $\left(V_{0}-M_{V}\right)=31.0$ is adopted. Our results demonstrate that nonunimodal globular cluster populations exist in low-luminosity ellipticals, as well as in giant ellipticals, and thus that the formation mechanisms for these galaxies may share some similarities.
\end{abstract}

Key words: galaxies: individual (NGC 1427) — galaxies: star clusters

\section{INTRODUCTION}

Globular clusters (hereafter GCs), being mostly very old systems, can provide a wealth of information about the early stages of galaxy formation. This fact has been recognized, discussed, and utilized for a long time (see, e.g., Searle \& Zinn 1978). The information is contained in the spatial distribution, chemical composition, and kinematic behavior of globular clusters within a galaxy.

One of the most interesting results of the past several years of research on the GC systems (GCSs) of elliptical galaxies is the discovery of multiple (typically bimodal) populations within a galaxy, as demonstrated by distinct metallicity, kinematics, and spatial distributions (see, e.g., Zepf \& Ashman 1993, Whitmore et al. 1995, Geisler, Lee, \& Kim 1996, Kissler-Patig \& Gebhardt 1998). Such multiple populations are found to be common in giant ellipticals (GEs; see, e.g., Gebhardt \& Kissler-Patig 1999 and Kundu 1999) and provide strong constraints on the galaxy formation process. Various scenarios have been put forward to attempt to explain the multiple formation epochs and/or mechanisms these populations demand. For example, Schweizer (1986) suggests that GCs could be formed in gaseous mergers, a scenario further developed by Ashman \&
Zepf (1992). Competing ideas currently include multiple epochs of in situ GC formation (see, e.g., Forbes, Brodie, \& Grillmair 1997) and accretion models such as those of Richtler (1995) or Côté, Marzke, \& West (1998).

Although both the volume and quality of the observational data have been consistently growing during recent years, most of these studies have been focused on GEs, primarily because of the larger data samples available, as the number of GCs in a galaxy generally corresponds directly to the luminosity or mass. It is clear, however, that any effort leading to the development of a comprehensive picture of the formation and evolution of GCSs, as well as that of the underlying stellar halos, must include not only these bright galaxies but also less luminous and less conspicuous systems, which are nonetheless more numerous.

NGC 1427, an elliptical galaxy (E3) in the Fornax cluster, falls into this last category, and its GCS has indeed been the subject of several previous papers. Forbes et al. (1996) carried out $V$ and $I$ photometry using the WFPC2 camera on the $H S T$ as part of a study that included a number of galaxies with kinematically distinct cores. Later, KisslerPatig et al. (1997) obtained independent observations using the same filters on the Las Campanas Observatory's $2.5 \mathrm{~m}$ 
telescope. Kundu (1999) has recently carried out a reinvestigation of the HST data as part of a large-scale study of GCSs using WFPC2 data.

These studies have turned the NGC 1427 GCS into the best-studied GCS in a low-luminosity elliptical (LLE). Here we adopt the terminology of Kissler-Patig, Forbes, \& Minniti (1998b), who defined an LLE as an elliptical with $M_{V}>-21 \mathrm{mag}\left(M_{V_{\mathrm{NGC1427}}}=-20.4 \mathrm{mag}\right.$ from the NED database). Interestingly, the Forbes et al. (1996) and KisslerPatig et al. (1997) studies found that the $V-I$ color distribution of the NGC 1427 GCS showed no clear evidence of nonunimodality. In addition, Kissler-Patig et al. (1998b) attempted to determine the constraints on models of galaxy formation via mergers using GCS data. In that paper, they discussed the NGC 1427 GCS in detail as an example of a unimodal distribution that would argue against the formation of LLEs in gaseous mergers (unless a particular combination of age and metallicity would make the GCS populations indistinguishable from each other). Gebhardt \& Kissler-Patig (1999) reduced WFPC2 $V$ - and $I$-filter data for the GCSs of 50 early-type galaxies, including a number of LLEs (not including NGC 1427), and concluded that, in general, the color distributions for the LLEs were "consistent with unimodality." In turn, Kundu (1999), based on the data used by Forbes et al. (1996), found that the $V-I$ color distribution of NGC 1427 is very likely bimodal. Indeed, in his study of color distributions of the GCSs in 29 ellipticals with a range of luminosity, he found evidence for bimodality in ellipticals throughout the entire luminosity range, from as faint as $M_{V}=-19.9 \mathrm{mag}$ up to $-22.7 \mathrm{mag}$.

Given the status of the NGC 1427 GCS as a leading example of an LLE GCS and the role it has played in developing ideas of galaxy formation, it is clearly important to clarify the observational picture. In such an approach, the critical factors are a good sample size, large radial coverage, and, especially, adequate metallicity sensitivity. Accurate metal abundances are required from a significant sample (hundreds) of GCs in a galaxy in order to resolve distinct metallicity peaks, if they exist, and a large radial coverage is needed to define any metallicity gradient.

Despite its popularity, especially for WFPC2 studies of stellar populations, $V-I$ unfortunately is only mildly metallicity-sensitive (see, e.g., Barmby et al. 2000). Nevertheless, all previous studies of the NGC 1427 GCS used it as their metallicity index. In addition, these studies covered only limited numbers of GCs, $\sim 150$ or less. This factor is especially problematic for LLEs, because they only possess relatively small GCSs. Finally, previous studies covered small areas of the galaxy. If different GC populations are present and their radial distributions are different, as often appears to be the case (see, e.g., Geisler et al. 1996), such data may be more sensitive to the presence of one or the other population given the small area imaged, and the resulting metallicity distribution will not be representative of the galaxy's entire GCS.

This paper aims at an improvement in our knowledge of the NGC 1427 GCS through a wide-field photometric study using the $C$ and $T_{1}$ filters of the Washington system (Canterna 1976). Geisler \& Forte (1990) and Forbes \& Forte (2001) have shown that the integrated $C-T_{1}$ color is twice as sensitive to metallicity as $V-I$. The new results can also be readily compared to those obtained for other galaxies using the same photometric system, e.g., NGC 1399 (Ostrov, Geisler, \& Forte 1993, hereafter OGF; Ostrov,
Forte, \& Geisler 1998, hereafter OFG) and NGC 4472 (Geisler et al. 1996, Lee, Kim, \& Geisler 1998). The metallicity calibration of the $C-T_{1}$ index has recently been substantially improved (Geisler \& Forte 2001). In addition, NGC 1427 is sufficiently nearby to allow the previously limited sample sizes to easily be doubled in a few hours of integration time using a $4 \mathrm{~m}$ telescope and the broadband Washington filters. Finally, the GCS of the entire galaxy can now be imaged in a single CCD frame with full spatial sampling.

\section{OBSERVATIONS AND DATA HANDLING}

\subsection{Observations}

CCD (Tek 2K No. 6) frames were obtained using the prime focus and the atmospheric dispersion corrector of the Blanco $4 \mathrm{~m}$ telescope at Cerro Tololo Inter-American Observatory (CTIO) during a night of service observing in 1997 October. Each frame is 14:7 on a side and has a pixel scale of 0.43 . The ARCON controller read out the chip through 2 amps with a readout noise of $\sim 3.6$ electrons. The data set consists of two $R_{\mathrm{KC}}(600$ and $1000 \mathrm{~s})$ and four $C$ (1500 s each) frames taken in seeing conditions of $\sim 1^{\prime \prime} 1$. Note that Geisler (1996) has shown that the $R_{\mathrm{KC}}$ filter is more efficient than the Washington $T_{1}$ filter and that $R_{\mathrm{KC}}$ and $T_{1}$ magnitudes are closely related, with only a very small color term and zero-point difference. In what follows we will use $T_{1}$ and $t_{1}$ in order to identify the standard and instrumental magnitudes, respectively, obtained with the $R_{\mathrm{KC}}$ filter (see below).

\subsection{Photometry}

We removed the smooth galaxy background from each frame by means of the routines contained in the VISTA image processing system. The photometry was performed using the DAOPHOT/ALLSTAR/ALLFRAME standalone suite of packages (Stetson 1987, 1991). We first derived a quadratic point-spread function (PSF), using 50-80 images per frame. Next, we used the MONTAGE2 program to obtain a combined image of all frames, in order to maximize $\mathrm{S} / \mathrm{N}$ for star finding, and then we ran a standard three-pass FIND/PHOT/ALLSTAR/SUBSTAR iteration on this frame. The output through the final ALLSTAR routine served as the input coordinate file for a single ALLFRAME reduction, which performed simultaneous photometry on all six frames. This yielded PSF photometry for 10,000 objects present in at least one $R_{\mathrm{KC}}$ image and three $C$ images (matching radius $=1$ pixel). Aperture corrections between the PSF radius and a radius of 15 pixels $(\sim 6$. .5$)$ were obtained using the PSF stars and were $\approx 0.0 \pm 0.02$ to 0.03 mag.

The link to the standard Washington system was set using observations of 70 standards from the list of Geisler (1996). These data yielded the following equations: $c=C$ $-0.035( \pm 0.019)-0.100( \pm 0.003)\left(C-T_{1}\right)+0.294( \pm 0.012)$ $X_{C}, \quad \mathrm{rms}=0.023$, and $t_{1}=T_{1}-0.711( \pm 0.019)-0.019$ $( \pm 0.003)\left(C-T_{1}\right)+0.100( \pm 0.012) X_{T_{1}}, \mathrm{rms}=0.019$, where $c$ and $t_{1}$ are the instrumental magnitudes measured through 15 pixel radius apertures (corrected to $1 \mathrm{~s}$ exposure time), $C$ and $T_{1}$ are the standard magnitudes, $C-T_{1}$ the standard color and $X_{C}$ and $X_{T_{1}}$ the respective air masses. These latter values were essentially 1.0 for the NGC 1427 observations. The night was clearly of good photometric quality.

After applying these transformations to the NGC 1427 photometry, we proceeded to cull the list in order to 
produce the cleanest sample of GC candidates possible. First, we removed spatially resolved objects. Note that at the distance of the Fornax cluster GCs appear as unresolved images on our frames. However, given our good seeing, we could eliminate most bright resolved objects with careful image classification criteria. Figure 1 shows, as an example, the output instrumental magnitude obtained from ALLFRAME versus the difference between that magnitude and the one derived from small-aperture photometry (4 pixel radius) for a given object. The position in this plane is used as a criterion to discriminate resolved objects. Unresolved objects at bright magnitudes show a small scatter about a constant small magnitude difference. The dispersion of the magnitude difference increases with magnitude as a result of the increasing measuring errors. In contrast, resolved objects appear with large negative values of this difference, as a consequence of their spatial structure.

That effect is clearly shown by the asymmetry of the distribution of the data points in Figure 1. A rejection envelope was then defined as 1.5 times the dispersion of the data points to the right of the modal value of the distribution of the magnitude differences (in a given ALLFRAME magnitude interval). This envelope was then mirrored to the left of the modal values. All objects outside these boundaries were rejected from the master list, as they were either resolved objects or suspected of large photometric errors. As a result of this culling procedure, 2083 objects remained.

\subsection{Errors and Completeness}

The photometric errors of the $T_{1}$ magnitudes and $C-T_{1}$ colors are shown in Figures 2 and 3, respectively. In order to keep the color errors below $\sim 0.1 \mathrm{mag}$ we restricted our analysis to objects brighter than $T_{1}=23.5 \mathrm{mag}$, i.e., some 0.3 mag fainter than the turnover magnitude observed in the GCS associated with NGC 1399 (see OFG), the dominant central cD galaxy in Fornax. Note that the errors for

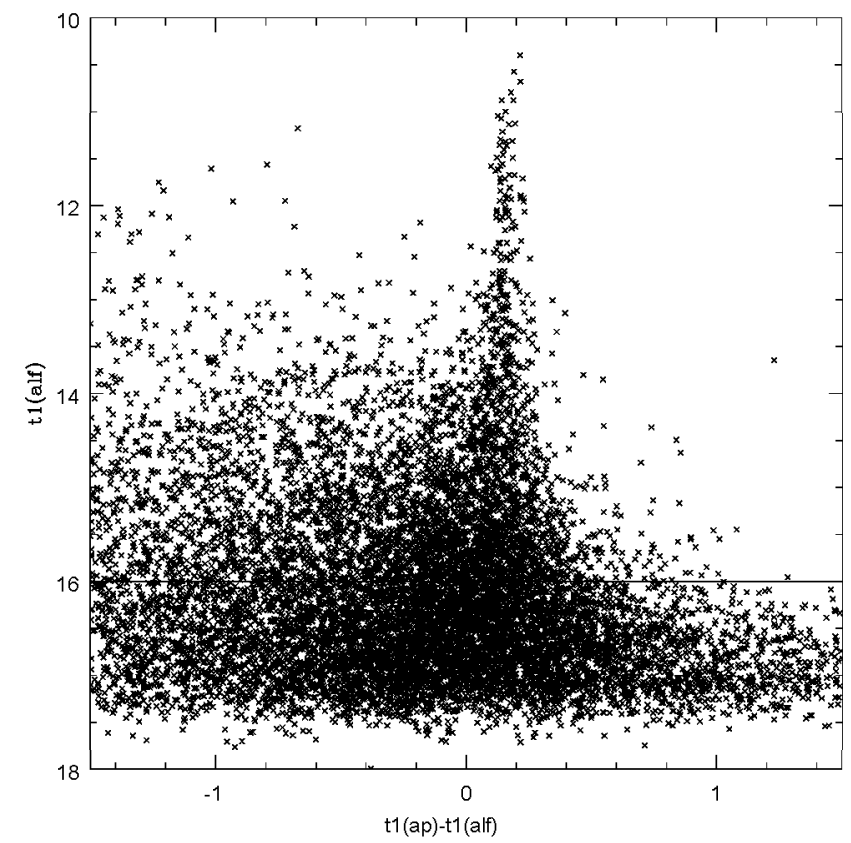

FIG. 1.-Instrumental ALLFRAME $t_{1}$ magnitude vs. the difference between the aperture magnitude (measured with a 4 pixel radius aperture) and the ALLFRAME magnitude, for all detected objects in the NGC 1427 field. The horizontal line is the limiting instrumental magnitude $T_{1}=23.5$.

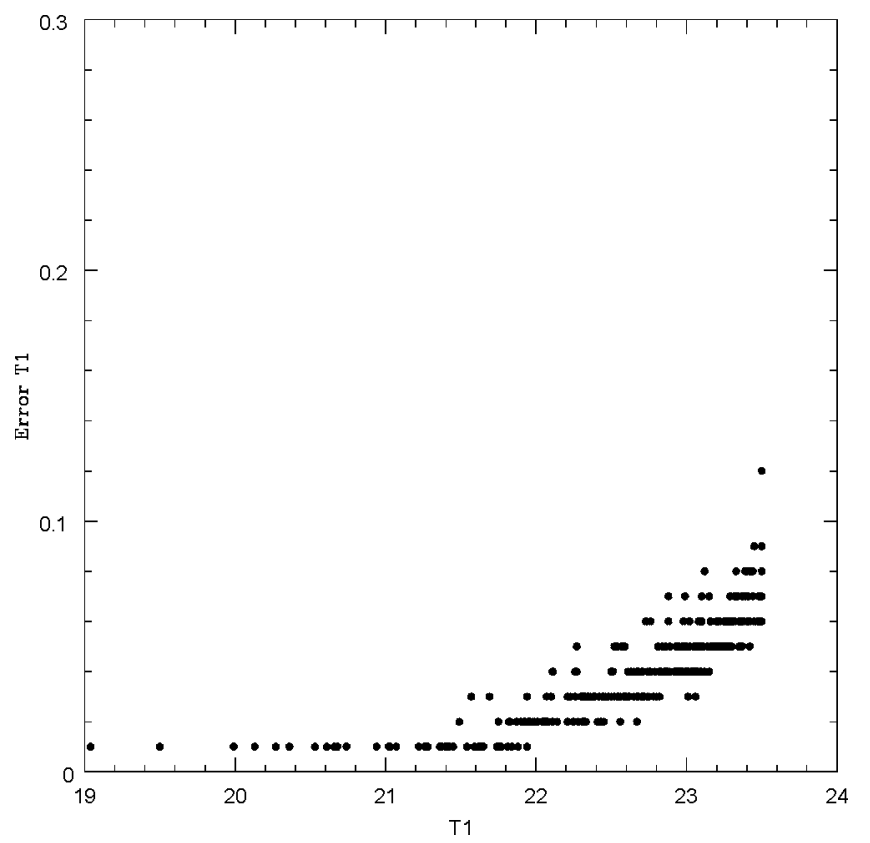

FIG. 2. $-T_{1}$ magnitude errors (approximated to $0.01 \mathrm{mag}$ ) as a function of $T_{1}$ for all the unresolved objects in the field of NGC 1427 brighter than $T_{1}=23.5$.

our selected sample are generally very small and that no further objects were discarded on the basis of their large errors at a given magnitude.

The completeness of the GC sample was ascertained through ADDSTAR experiments. These experiments show that, at a limiting magnitude $T_{1}=23.5$ and for objects with colors as red as $C-T_{1}=1.50$, the overall completeness of the survey is close to $95 \%$. This level decreases to $85 \%$ if the color range is extended up to 2.0 in $C-T_{1}$.

The $T_{1}$ versus $C-T_{1}$ color-magnitude diagram for all unresolved objects is depicted in Figure 4, where the horizontal line is our adopted limiting $T_{1}$ magnitude. The upper

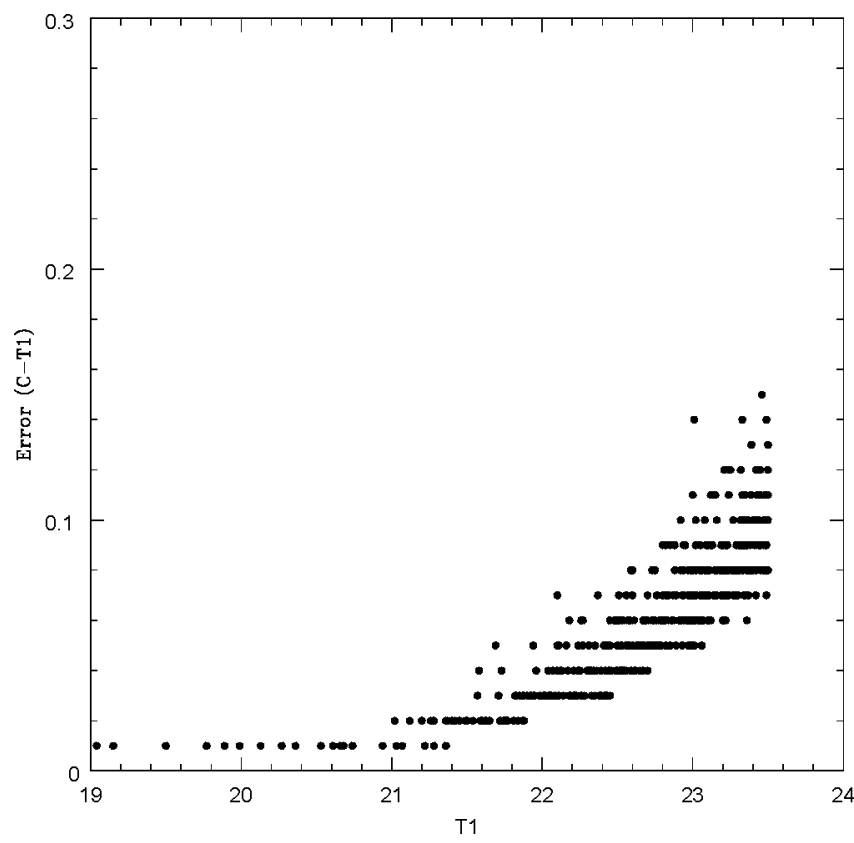

FIG. 3. $-C-T_{1}$ color errors as a function of $T_{1}$ magnitude for the same objects as in Fig. 2 . 


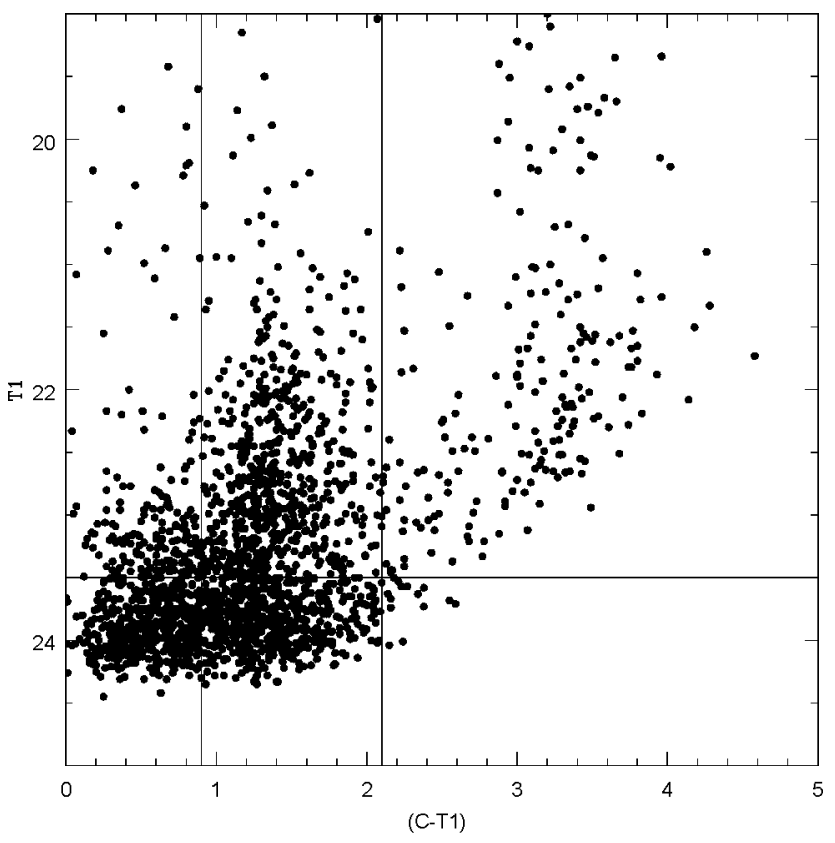

FIG. 4. $-T_{1}$ magnitude vs. $C-T_{1}$ color for unresolved objects in the field of NGC 1427. The horizontal line represents the selected limiting magnitude of this study. The vertical lines at $C-T_{1}=0.9$ and 2.1 define the expected domain of the globular clusters associated with NGC 1427.

magnitude limit was taken as $19.0 \mathrm{mag}$, based on a study of the more populous NGC 1399 GCS (OGF; OFG). The vertical lines at $C-T_{1}=0.90$ and 2.10 show our adopted definition of the color domain of the GCs. This assumption comes from the observed situation in a number of galaxies that contain old GCSs (e.g., the Milky Way, NGC 4472,

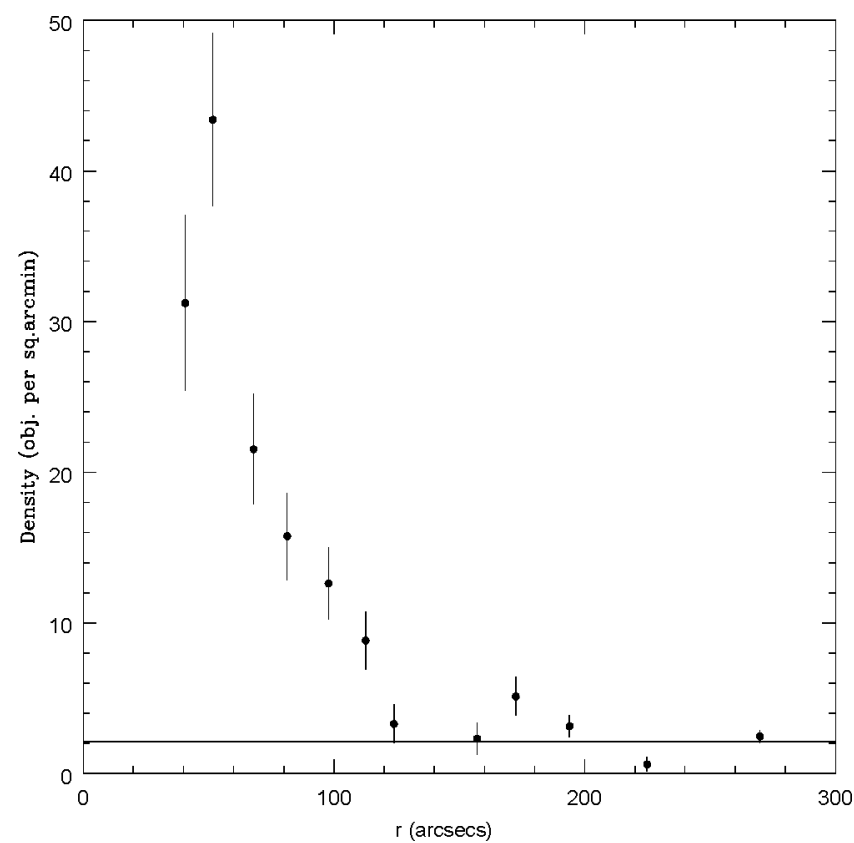

FIG. 5.-Background-corrected areal density (objects per square arcminute) of all the unresolved objects as a function of apparent galactocentric distance. Error bars come from Poissonian statistics (including the effect of background-level uncertainty). Background level is indicated by the horizontal line. This sample includes GC candidates with $T_{1}$ from 19.0 to 23.5 and $C-T_{1}$ from 0.9 to 2.1 . The first bin shows the effect of incompleteness.

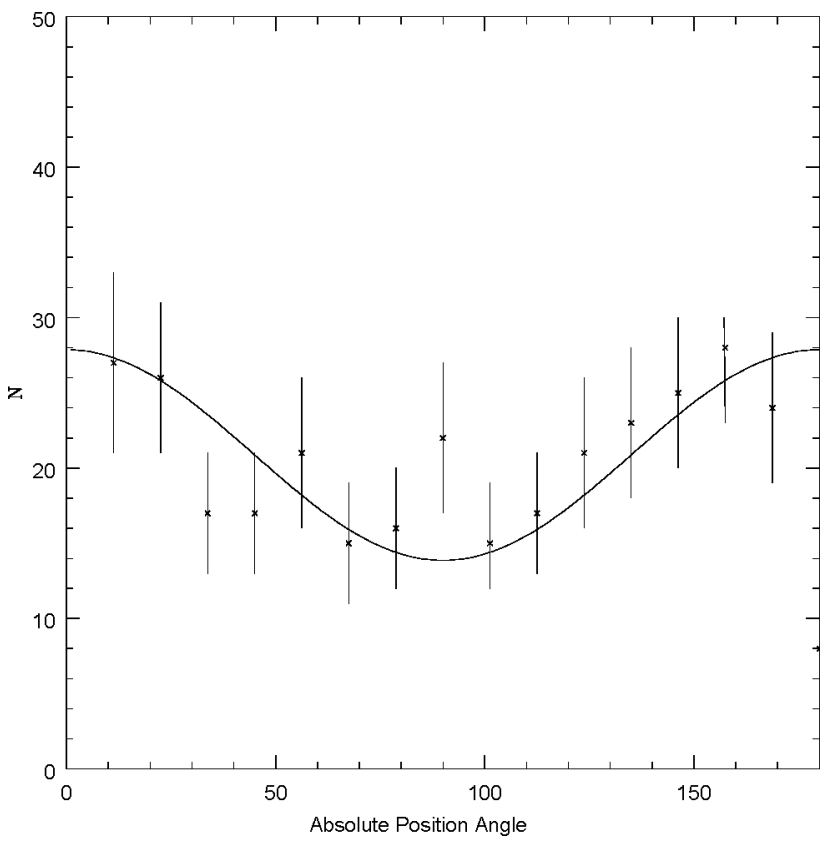

FIG. 6. - Azimuthal number of unresolved objects (in sectors of 22.5) as a function of the absolute value of the position angle measured with respect to the galaxy major axis (see $\$ 3$ ).

NGC 4486). Note that, in what follows, we have assumed zero reddening for NGC 1427, on the basis of the $C M T_{1}$ photometry discussed by OFG. A total of 328 unresolved objects fall within these color and magnitude limits and a galactocentric radius from $30^{\prime \prime}$ to $300^{\prime \prime}$ (see below) and are considered GC candidates. The remaining analysis will be

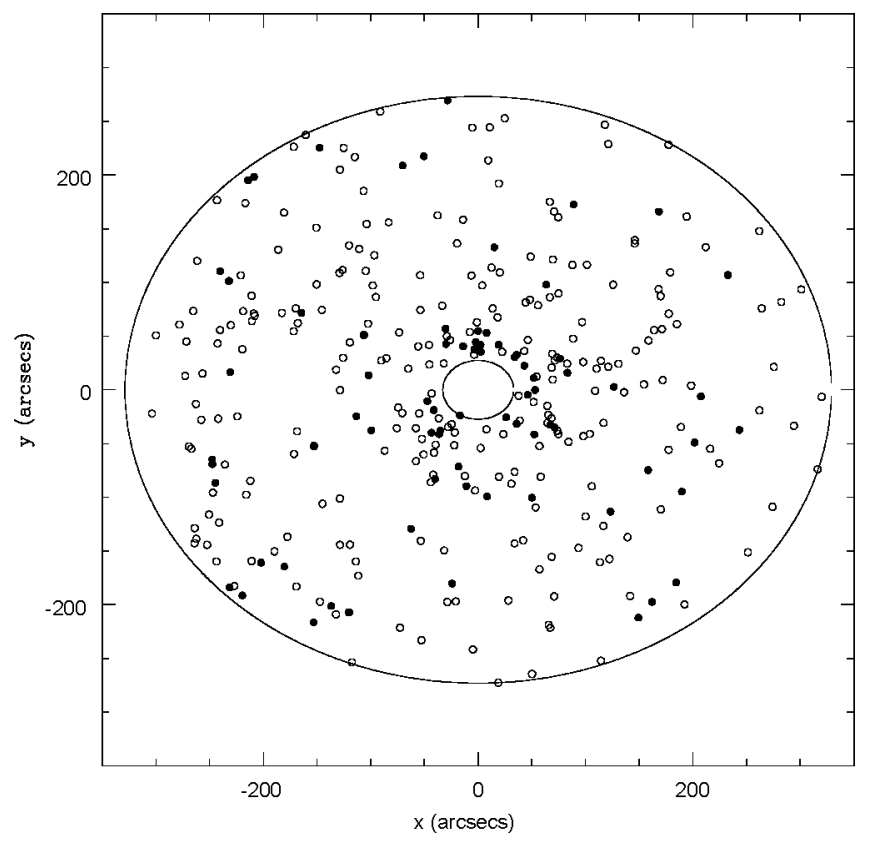

Fig. 7.-Rectangular $(x, y)$ coordinates (in a system defined by the galaxy axis) of the 328 unresolved objects detected $30^{\prime \prime}-300^{\prime \prime}$ in elliptical pseudoradius $\left(r_{e}\right)$ from the NGC 1427 center and inside the globular cluster color-magnitude domain of the survey. The ellipses have ellipticities of 0.83 . Open circles: $\mathrm{GC}$ candidates bluer than $C-T_{1}=1.6$; filled circles: those redder than that color. 
conducted only on these candidates. The photometry for these objects can be obtained from the first author upon request.

\subsection{Field Contamination}

Because NGC 1427 is an LLE, its GCS is not very populous, and the determination of the total number of clusters, as well as that of their spatial distribution, requires a good estimate of the background contamination arising from unresolved objects along the line of sight. For this purpose, we defined a comparison area composed of two strips along the entire southern and eastern edges of the CCD frames (i.e., farthest from the galaxy), with widths of $3: 7$ and 1.9 , respectively. The total area is $77.7 \mathrm{arcmin}^{2}$, equal to that in which the GCS survey was performed, and it contains 163 objects in the adopted color-magnitude domain of the GCs. This corresponds to a background surface density of $2.1 \pm 0.2 \operatorname{arcmin}^{-2}$.

\section{SPATIAL DISTRIBUTION OF THE GLOBULAR CLUSTER CANDIDATES}

As a first step in determining both the extent and shape of the GCS, we obtained the surface density run with respect to galactocentric radius for the GC candidates within circular annuli, as shown in Figure 5. This curve, which does not include the inner $30^{\prime \prime}$, where the $T_{1}$ images are saturated, suggests that the boundary of the system is between $250^{\prime \prime}$ and $300^{\prime \prime}$ from the galaxy center. An estimate of the GCS's shape was then performed by analyzing the azimuthal distribution of the GC candidates. First, the orientation of the galaxy's major axis was determined from the isophotes, yielding a position angle of $78^{\circ} \pm 2^{\circ}$. Then, each GC candidate was counted within a moving bin $22^{\circ} .5$ wide (and shifted in steps of 11.25 ), covering a galactocentric range from $30^{\prime \prime}$ to $300^{\prime \prime}$. The distribution was folded, in order to increase the S/N of the counts, and only the absolute position angle with respect to the eastern semimajor axis was considered in defining a given angular bin.

The resulting azimuthal distribution of the GC candidates is shown in Figure 6. In order to derive the GCS's flattening we left out the $240^{\prime \prime}-300^{\prime \prime}$ region, where field contamination becomes dominant. The method of coordinate moments, described by McLaughlin, Harris, \& Hanes (1994) and used by those authors to estimate the M87 GCS's flattening, was then applied to this revised data set (containing 241 objects), yielding a flattening of $b / a=0.83 \pm 0.05$ and a position angle similar to that of the isophotal light $\pm 20^{\circ}$. In what follows, we adopt $b / a=$ 0.83 as the GCS's overall flattening, in order to compute the galactocentric pseudoradius, $\boldsymbol{r}_{\boldsymbol{e}}=(a b)^{0.5}$, for each object.

Figure 7 shows the distribution of the 328 unresolved objects within $30^{\prime \prime}-300^{\prime \prime}$ in pseudoradius from the galaxy center (and contained in the adopted color-magnitude domain of the GCs) in a coordinate system defined by the galaxy axis. Different symbols are used for blue and red GCs (see below).

\section{GLOBULAR CLUSTER MAGNITUDE AND COLOR DISTRIBUTION}

The $T_{1}$ magnitudes and $C-T_{1}$ colors for all unresolved objects in the galactocentric range from $30^{\prime \prime}$ to $300^{\prime \prime}$ are

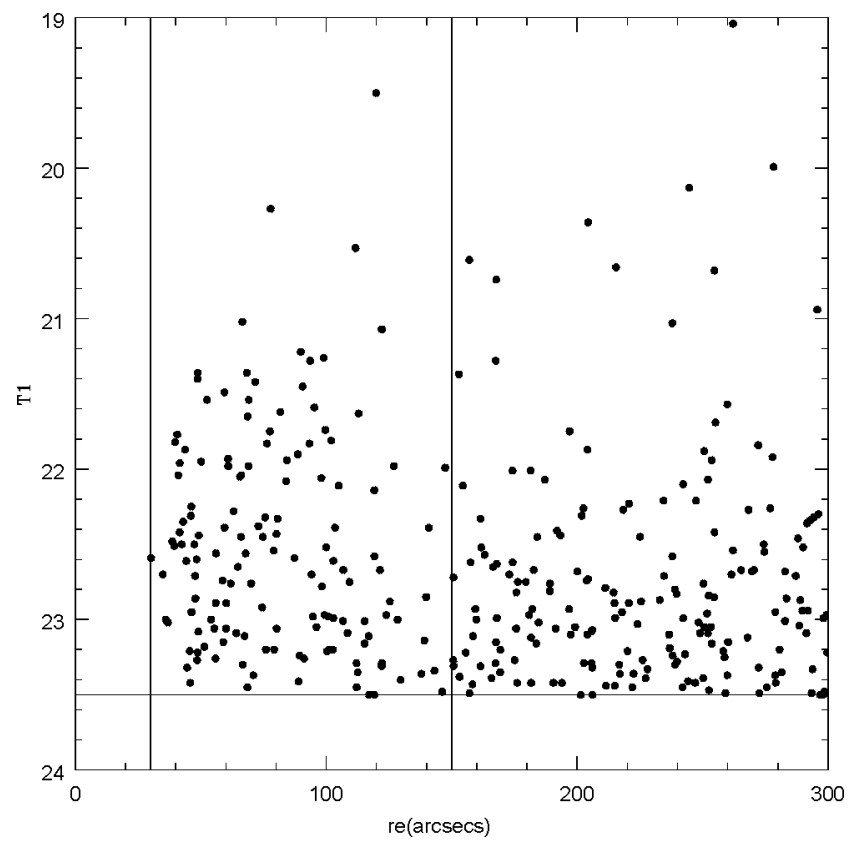

FIG. 8. $-T_{1}$ magnitudes vs. pseudogalactocentric radius for all objects depicted in Fig. 7. Vertical lines at $30^{\prime \prime}$ and $180^{\prime \prime}$ define the GC sample that, after background correction, was used to derive the integrated luminosity function.

shown in Figures 8 and 9, respectively. This sample includes more than half of the total GC population in NGC 1427 (see $\S 6$ ). However, the total contribution from background objects in this area represents some $50 \%$ of the whole data set. This contribution must be statistically removed in order for us to derive both the luminosity function and the intrinsic color distribution of the GCS. Figure 10 shows the $T_{1}$ luminosity function for unresolved objects that are both in

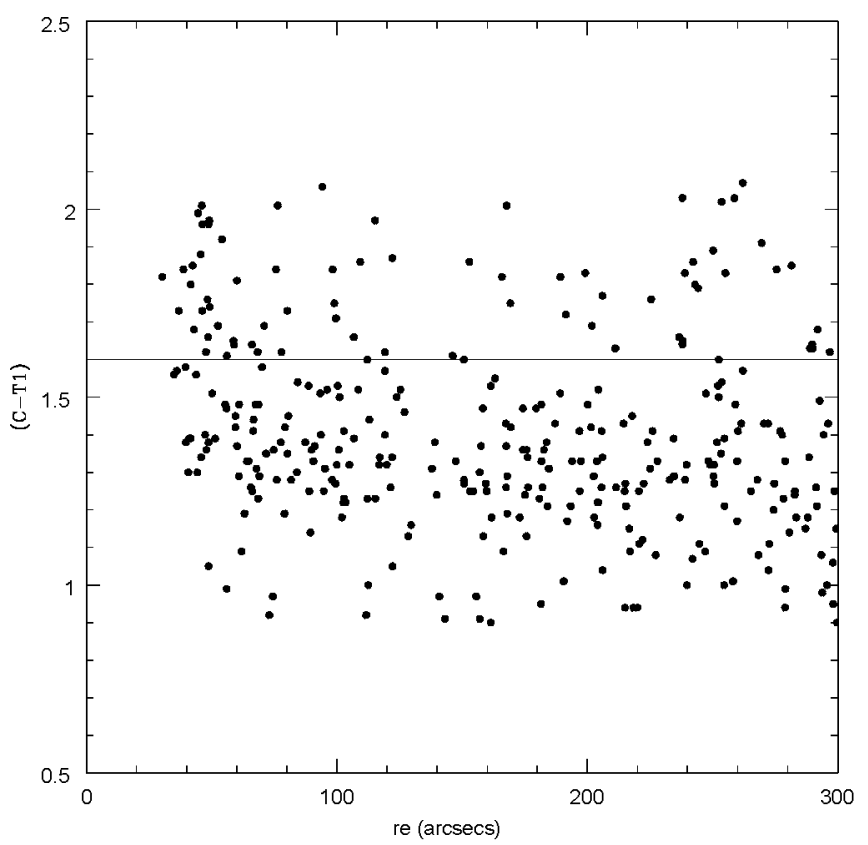

FIG. 9. - Same as Fig. 8, but for $C-T_{1}$ color. The horizontal line is the adopted boundary between red and blue globular clusters. 


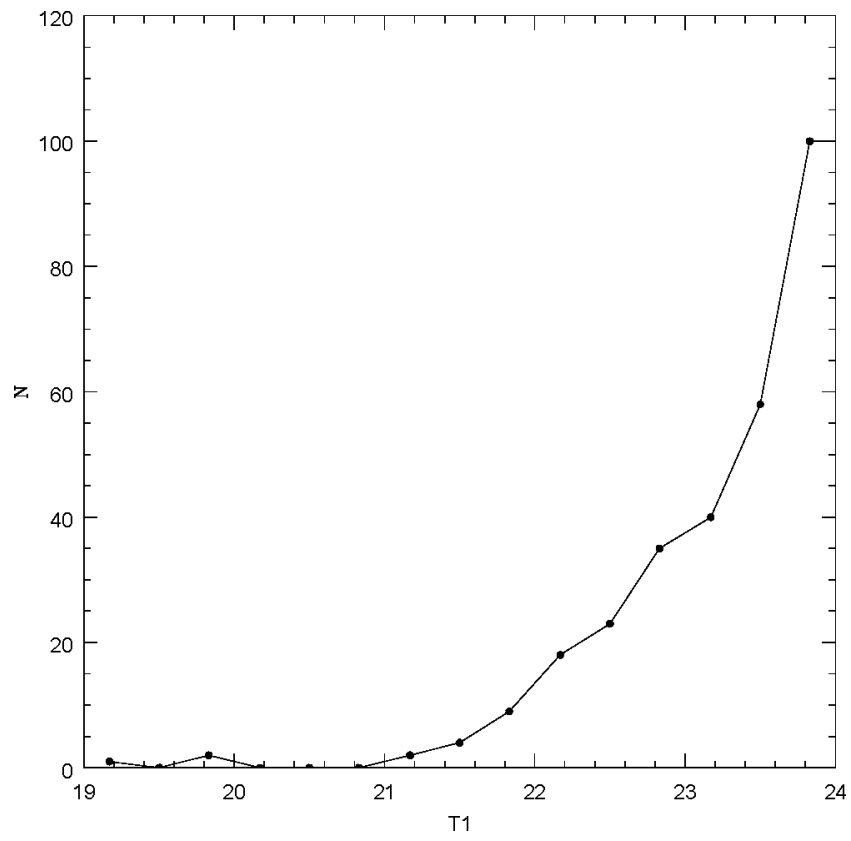

FIG. 10.-Luminosity function of unresolved objects in the 77.7 $\operatorname{arcmin}^{2}$ comparison field as a function of the $T_{1}$ magnitude (in $0.33 \mathrm{mag}$ bins).

the comparison area and in the color-magnitude domain of the GCs. This ensemble shows a steep increase for $T_{1}>23$, and an inspection of Figure 4 reveals that most of them are rather blue objects. In order to decrease the effect of the statistical noise of this population on the net integrated luminosity function of the GCs, the analysis was restricted to an elliptical area bounded between $30^{\prime \prime}$ and $180^{\prime \prime}$ in pseudoradius. The resulting distribution of GCs by $T_{1}$ is depicted in Figure 11. This luminosity function includes the photometric completeness corrections as a function of color, mentioned in $\S 2.3$.

The relatively small GC population and the associated statistical uncertainty prevent a meaningful determination of the position of the turnover. As an indicative fit, Figure 11 includes a Gaussian curve characterized by a dispersion of $1.2 \mathrm{mag}$ and centered at $T_{1}=23.3 \mathrm{mag}$; these parameters are consistent with the values for the NGC 1399 GCS given in OFG and will be used later in the estimate of the specific frequency $\left(S_{N}\right)$ of GCs, as defined by Harris \& van den Bergh (1981). We note, however, that the NGC 1399 turnover comes from a GC sample (clusters between $30^{\prime \prime}$ and $120^{\prime \prime}$ from the galaxy center) with an average $C-T_{1}$ color of 1.63 , i.e., 0.21 mag redder than that of the NGC 1427 GCs in our sample. This color difference means that the NGC 1427 GCs are some 0.5 dex more metal poor than those of NGC 1399 and, according to the models given by Ashman, Conti, $\&$ Zepf (1995), this would make the turnover some $0.13 \mathrm{mag}$ brighter than that of NGC 1399. This effect on the estimate of the total GC population and the one arising from the adopted distance modulus are discussed in $\$ 6$.

An accurate assessment of the NGC 1427 GC color distribution is the main goal of this study. In order to properly investigate this issue, the effect of background contamination must again be addressed. A generalized color histogram representative of the background population was obtained, using the comparison-region data, by convolving

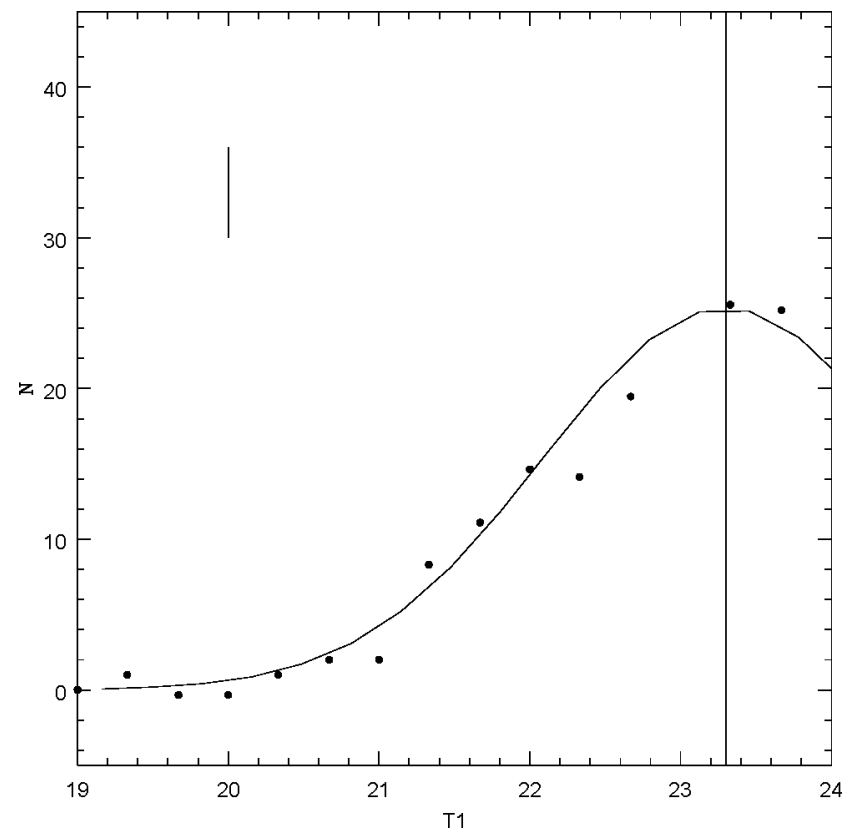

FIG. 11.- Luminosity function for the globular clusters associated with NGC 1427 (after subtraction of the field contamination). The Gaussian fit corresponds to a turnover at $T_{1}=23.3 \mathrm{mag}$ and a dispersion of $1.20 \mathrm{mag}$. The bar at the upper left shows typical Poissonian fluctuation expected at the peak of the curve.

each observed color with a Gaussian with a dispersion of $0.07 \mathrm{mag}$, a value comparable to the overall color error in our sample. The generalized histogram for the comparison region is depicted in Figure 12. This smoothed background color distribution was then subtracted from a similar histogram obtained for all the GC candidates in the $30^{\prime \prime}-300^{\prime \prime}$ region, leading to the corrected color distribution displayed

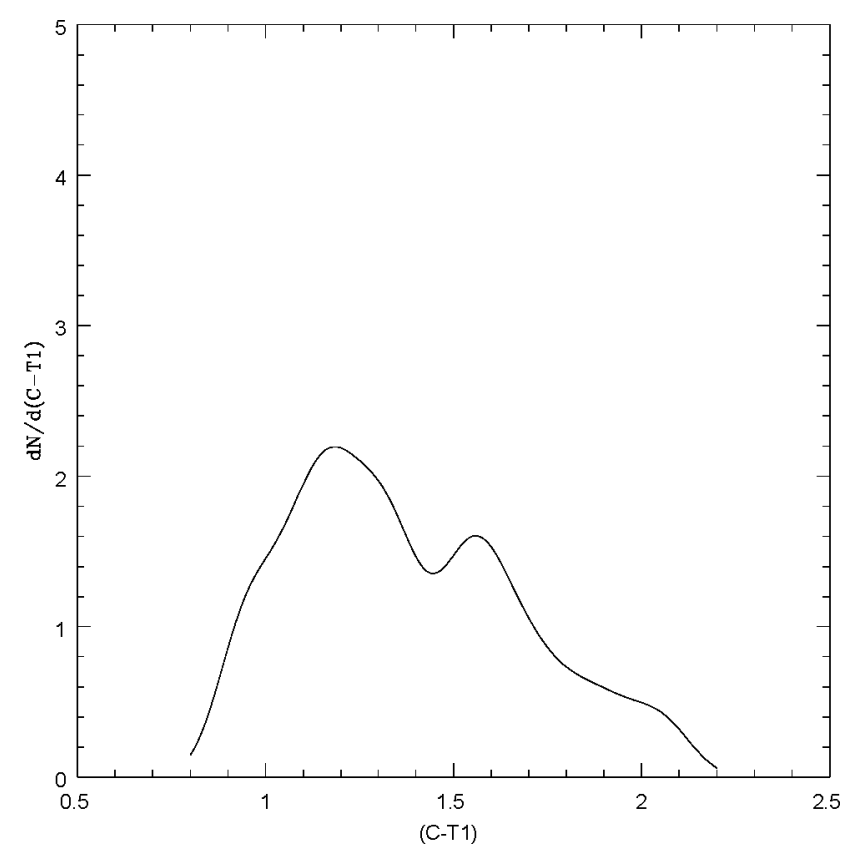

FIG. 12.-Generalized $C-T_{1}$ color histogram for unresolved objects in the comparison field. 


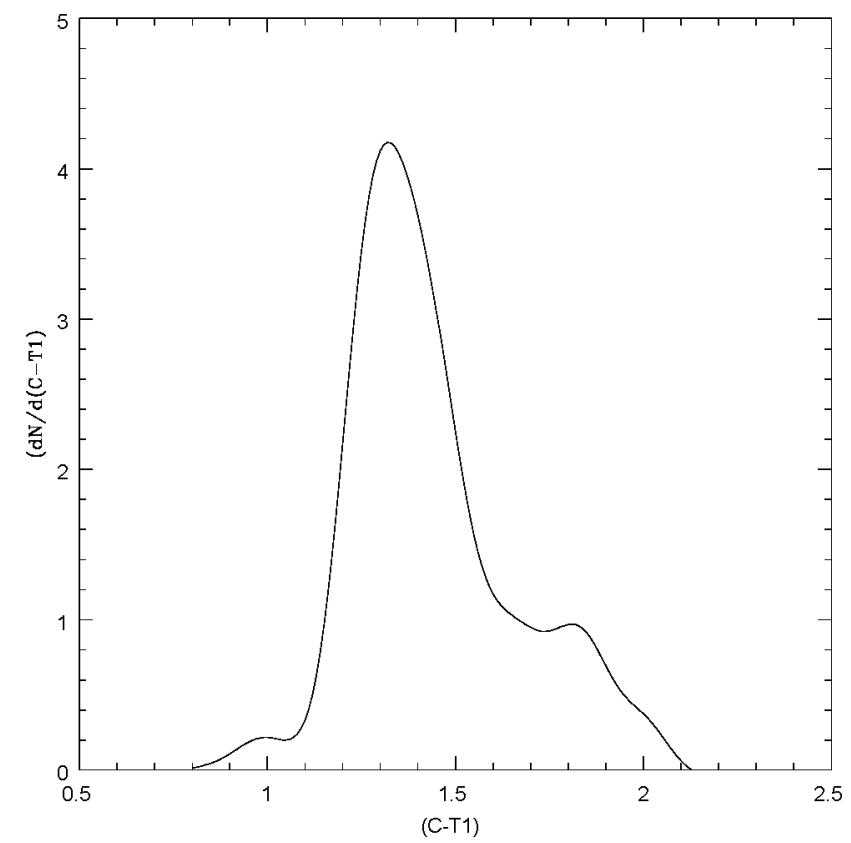

FIG. 13.- Same as Fig. 12, but for globular cluster candidates associated with NGC 1427 after correcting for field contamination. The bimodal distribution is borne out by the statistical tests described in the text.

in Figure 13. This last diagram reveals the existence of two separated peaks, strongly suggesting bimodality.

In order to quantitatively assess this visual impression, we analyzed this distribution using the NGAUSSFIT routine in STDAS. We compared a single-Gaussian fit to a double-Gaussian one. Although this clearly involves an important assumption, viz., that the color distribution is well represented by a Gaussian (or Gaussians), the assumption appears reasonable, given its suitability for other wellstudied GCSs (e.g., the Galaxy, Armandroff \& Zinn 1988; NGC 4472, Geisler et al. 1996). The double-Gaussian fit yields a $\chi^{2}$-value 10 times smaller than that for a singleGaussian fit, and it yields an rms 3 times smaller. In addition, a single-Gaussian fit severely underestimates the number of clusters with $C-T_{1}$ redder than 1.6 and is a poor representation of the data. The two-Gaussian fit returns values of $C-T_{1}=1.35$ and 1.73 for the means of the two distributions, with $\sigma=0.12 \mathrm{mag}$ (this value was determined using only the much larger blue sample and then fixed for the red sample) and a ratio of blue to red objects of 3.9 .

We also performed a KMM test on a backgroundcorrected sample. The background correction was performed by deleting GC candidates closest in color to objects in the comparison region list (color difference always less than the typical color error). This test returns a probability less than 0.001 that a single-Gaussian fit is preferred to a double-Gaussian. KMM identifies two groups, centered at $C-T_{1}=1.35$ and 1.79 , with a common $\sigma=0.15 \mathrm{mag}$ and a blue-to-red ratio of 4.4 , in very good agreement with the NGAUSSFIT results. The number of objects in the red group is 32 , in a total population of 174 GCs.

The dispersions of those fits include both the contribution of the observing errors and that of the smoothing kernels. After removing these effects, and using the colormetallicity relation given by Geisler \& Forte (1990), we determined that the metallicity dispersion of the GC population is $0.16-0.26$ in $[\mathrm{Fe} / \mathrm{H}]$.

Finally, we also used the BOOTSTAT package as implemented by Gebhardt \& Kissler-Patig 1999, kindly provided by M. Kissler-Patig. The DIP statistic calculated by this package is yet another indicator of the unimodality of a distribution. For our background-corrected sample of GC candidates, BOOTSTAT returned a DIP statistic of 0.5 with upper and lower $1 \sigma$ confidence limits of 0.79 and 0.50 . This technically signifies that there is only a $50 \%$ probability that the distribution is not unimodal. However, 0.5 is the minimum value and means that the test is uncertain.

Given the statistical tests that we performed, we are led to conclude at a very high confidence level that the NGC 1427 GCS color distribution is bimodal. Throughout the rest of this paper, we will refer to blue GCs (BGCs), defined as all GC candidates with $0.9<C-T_{1}<1.60$, and red GCs (RGCs), with $1.60<C-T_{1}<2.1$. Assuming that these samples are described by the two Gaussians derived above, splitting them at this color means that each population is contaminated by the other by no more than $10 \%$.

Further support that this simple division by color really separates two GC populations is provided by the distinct radial dependences of the RGCs and BGCs. As shown in Figure 7, where each population is shown with a different symbol, the red GCs appear much more concentrated than the blue ones. In fact, when the background contamination expected for the RGCs is removed, this family practically disappears beyond a galactocentric radius of $120^{\prime \prime}$. We will discuss this conclusion and its consequences further in $\S 7$.

\section{COMPARISON OF GCS AND GALAXY HALO COLORS}

The mean colors of the GCS appear to be bluer, in general, than those of their underlying galactic halos (Forte, Strom, \& Strom 1981). In this paper, we split the analysis in terms of the BGC and RGC populations.

Figure 14 shows the color gradient of the BGCs. In order to analyze the behavior of their $C-T_{1}$ colors, as a function of galactocentric radius, the clusters were grouped in annular regions with $30^{\prime \prime}$ widths, corrected for background contamination using smoothed color distributions similar to those described above, and the modal color was adopted as representative for each annulus. A least-squares fit to these modal colors leads to $\Delta\left(C-T_{1}\right) / \Delta \log \left(r_{e}\right)=-0.09$ \pm 0.03 , where $C-T_{1}$ is in magnitudes and $r_{e}$ is in arcseconds.

A $C-T_{1}$ color profile for the NGC 1427 galaxy halo was obtained by combining the smooth galaxy images delivered by the VISTA processing package (and used to remove the galaxy light before performing the DAOPHOT photometry). This profile and that of NGC 1399 (Forte, Pellizza, \& Faifer 2001) are also depicted in Figure 14. Both galaxy profiles seem somewhat steeper than, but comparable to, the BGCs' color trend. The BGCs' color gradient, once turned into a metallicity gradient (e.g., using the relation given in Geisler \& Forte 1990), becomes $\Delta[\mathrm{Fe} / \mathrm{H}] /$ $\Delta \log (r)=-0.21 \pm 0.05$ ( $r$ in kiloparsecs), a value that is readily comparable to the average gradient derived by Davies, Sadler, \& Peletier (1993) for a number of early-type galaxy halos.

Figure 14 also includes a straight line that results from a fit to the modal colors of BGCs in the periphery of NGC 1399 (from a data set obtained during the same run and treated in a similar way; Geisler \& Forte 2001). The slope of 


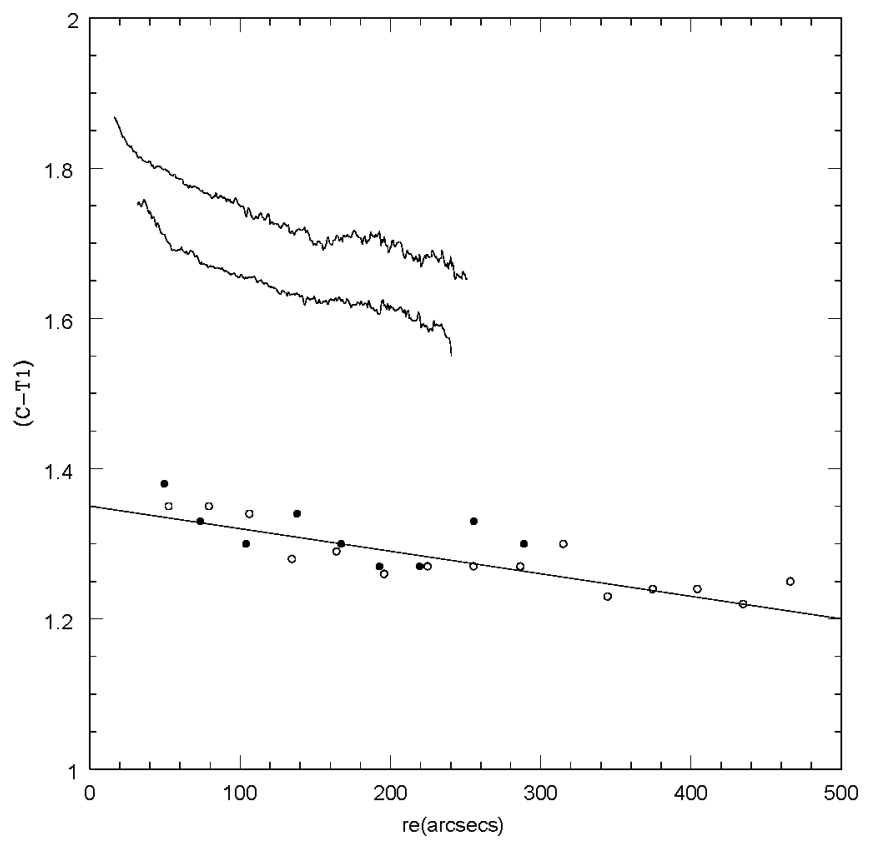

FIG. 14. $-C-T_{1}$ color gradient for the blue globular cluster population in NGC 1427 (filled circles) and NGC 1399 (open circles). The straight line is a fit to the latter values. The galaxy halo color gradients for NGC 1399 (top curve) and NGC 1427 are also shown.

this line, determined over a galactocentric range twice as large as the one covered by the BGCs in NGC 1427, is $\Delta\left(C-T_{1}\right) / \Delta \log \left(r_{e}\right)=-0.11 \pm 0.02\left(C-T_{1}\right.$ in magnitudes and $r_{e}$ in arcseconds). It is remarkable that, despite both the morphological and integrated brightness differences between NGC 1427 and NGC 1399, the color gradients of the BGCs in each galaxy appear to be equal within the uncertainties.

The case of the red clusters is not so clearly defined, because their number is relatively small and, after the subtraction of the expected background contribution, they cover a reduced range in galactocentric radius. We note that the mean color of this cluster population (derived from both the Gaussian fit and the KMM analysis) is very similar to that of the galaxy halo in the innermost regions, a situation that also holds for a number of other elliptical galaxies (see, e.g., Geisler et al. 1996, OFG, and, for a more recent discussion, Forbes \& Forte 2001).

\section{THE TOTAL GC POPULATION AND SPECIFIC FREQUENCY}

The number of globular clusters can be estimated using the results obtained in this paper, under the assumption that the luminosity function is fully Gaussian. For the inner $60^{\prime \prime}$, however, we adopt the surface GC density derived by Forbes et al. (1996) using HST images, which after areal integration yields a total of $270 \pm 30$. For the outer region, from $60^{\prime \prime}$ to $300^{\prime \prime}$, we start with the turnover magnitude $T_{1}=23.24$ obtained by OFG for NGC 1399, and, in accord with the metallicity effects discussed in $\S 4$ that would make the NGC 1427 GCS turnover 0.13 mag brighter, we finally adopt a turnover at $T_{1}=23.11$. In what follows, the quoted uncertainties come from counting statistics. Down to $T 1=23.5 \mathrm{mag}, 234$ objects were detected, and after removing 123 objects expected from the comparison-field sta- tistics, a total of $122 \pm 20 \mathrm{GC}$ candidates are left. Then, we correct for photometric completeness (1.11, corresponding to $90 \%$ completeness) and for the fraction of unsampled luminosity function (39\%), leading to a total of $201 \pm 35$ GCs. This number, added to that for the inner region, yields a total GC population of about $470 \pm 40$, in very good agreement with the estimate obtained by Kissler-Patig et al. (1997) $(510 \pm 87)$.

The total areal GC density as a function of galactocentric radius from both Forbes et al. (1996) and this work, properly scaled, as well as the $T_{1}$ surface brightness for the galaxy halo, is depicted in Figure 15. This diagram shows that the areal density of GCs is appreciably shallower than that of the halo light. A fit of the form density $\approx r^{-\alpha}$ yields $\alpha=2.0 \pm 0.2$, whereas for the $T_{1}$ halo light $\alpha=2.35 \pm 0.1$. We note that the GC density run obtained in this work agrees with that derived by Kissler-Patig et al. (1997), although these authors argued that both the density profile and galaxy halo light have the same slope.

In order to derive the specific frequency of GCs, we first integrate the galaxy $T_{1}$ brightness profile out to a galactocentric radius of $300^{\prime \prime}$, which yields $T_{1}=10.41 \pm 0.10$. This magnitude can be transformed into a visual one by using the color-color relations discussed by Forbes \& Forte (2001). These relations and the adoption of $C-T_{1}=1.70$ as a representative (luminosity-weighted) color for the galaxy halo yield $V-T_{1}=0.55$ and thus $V=10.96$.

If a distance modulus to Fornax $\left(V_{0}-M_{v}\right)=31.0$ is adopted (from OFG), the resulting integrated absolute magnitude is $M_{V}=-20.05$. Combining the estimates of the total cluster population and galaxy brightness inside $300^{\prime \prime}$ (or $23 \mathrm{kpc}$ ) from the galaxy center leads to an overall spe-

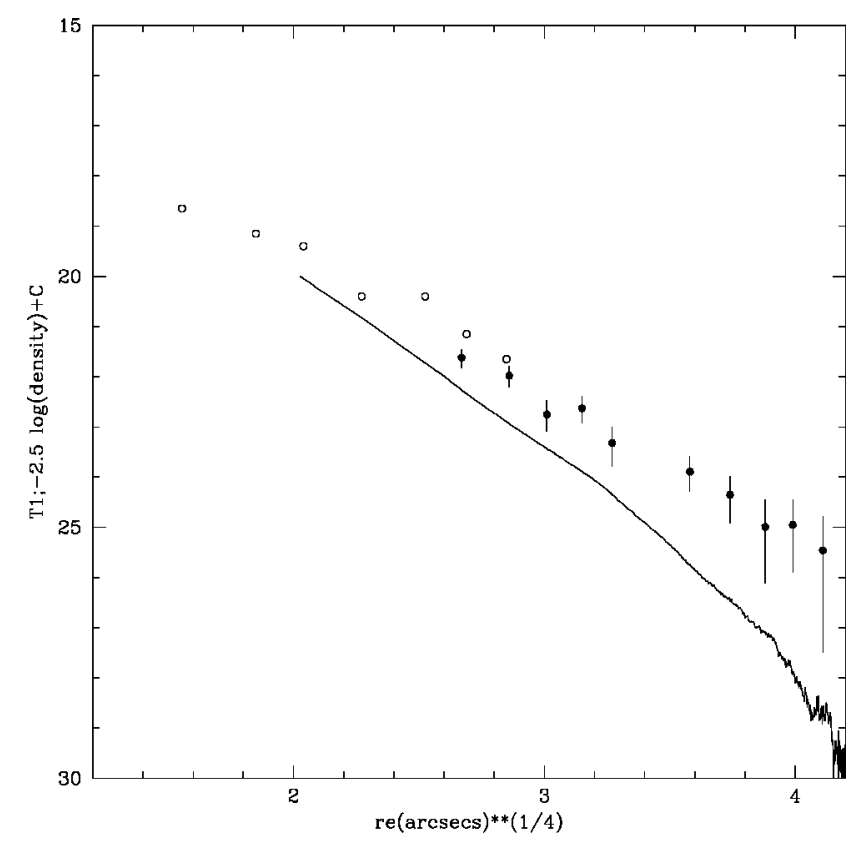

FIG. 15.-NGC $1427 T_{1}$ surface brightness as a function of pseudoradius. Filled circles show the density of globular clusters obtained in this work after correcting for observational and sampling completeness, in a scale defined as $-2.5 \log$ (density) $+C$; where $C=25.2$ is an arbitrary constant. Open circles show values from the density curve derived by Forbes et al. (1996) from HST observations. The uncertainty in the galaxy light profile is estimated at $\pm 0.1 \mathrm{mag}$ and $\pm 0.8 \mathrm{mag}$ at $r_{e}^{0.25}$ of 3.2 and 4.0 , respectively. 
cific cluster frequency $S_{N}$ of $4.5 \pm 0.8$. In fact, the different GC density and halo light slopes imply that $S_{N}$ varies with galactocentric radius. In comparison, the adoption of the distance to Fornax derived from HST observations of Cepheid stars, i.e., $(m-M)_{V}=31.3 \pm 0.1$ (Prosser et al. 1999; Madore et al. 1999), implies $S_{N}=3.4 \pm 0.6$. The range of $S_{N}$ values obtained for the NGC 1427 GCS is not far from the specific frequency values quoted for normal ellipticals, i.e., nonbright cluster galaxies. For example, Harris, Harris, \& McLaughlin (1998) give $S_{N}=3.3 \pm 0.2$ for these galaxies.

\section{DISCUSSION}

\subsection{Color Bimodality}

NGC 1427 possesses a bimodal GC population, with two cluster families having distinct metallicity and spatial distributions. Several previous studies obtained discrepant results regarding the shape of the metallicity distribution compared with those in this paper. The difference probably arises from their poorer metallicity sensitivity and smaller sample size. Both the Forbes et al. (1996) and Kissler-Patig et al. (1997) data sets have typical errors $\sigma(V-I) \sim 0.1$ or larger. In turn, our median $C-T_{1}$ color error is 0.07 . Given that the $C-T_{1}$ index is twice as metallicity-sensitive as $V-I$, it follows that our metallicity resolution enjoys an advantage over these previous studies of a factor close to 3 . Such an advantage is clearly crucial in resolving a metallicity distribution such as that displayed by the NGC 1427 GCS, where one peak is strongly dominant and the number of RGCs is small. The color error for our faintest GC is still comparable with that for a typical cluster in the studies based on the $V-I$ color. We also note that the two color peaks are separated by about $0.2 \mathrm{mag}$ in $V-I$, which is only twice the typical error of the Forbes et al. (1996) and Kissler-Patig et al. (1997) studies.

The GC sample size is also of importance, especially given the small number of RGCs in NGC 1427. A possible illustration of this is the interesting result noted by KisslerPatig et al. (1997) that, although they found no evidence of bimodality in the individual GCSs of NGC 1427 (note that they had only 76 GC candidates) and three other Fornax LLEs, bimodality appeared when all four color distributions were plotted together. When the combined color peaks obtained by these authors- $V-I \sim 1.02$ and $1.18-$ are transformed to $C-T_{1}$ using the color-color relations given by Forbes \& Forte (2001), they yield values of 1.40 and 1.72 . These values agree to within $\pm 0.05 \mathrm{mag}$ with the mean colors obtained in the present study for the NGC 1427 peaks, more evidence that this GC population is bimodal.

We note that Kundu (1999) finds a much higher proportion of RGCs to BGCs in his sample (roughly equal numbers) compared with our result. This situation may arise from the fact that WFPC2 observations cover only a small, central part of the entire GCS, while our data cover a much larger area but avoid the innermost region (because of saturation). Since the RGCs are much more strongly concentrated than the BGCs, the WFPC2 data sample a much higher fraction of RGCs than our data.

We are less certain why Kundu (1999) was able to discern bimodality in his reduction of the Forbes et al. (1996) data set. The number of GCs is very similar for both cases. Kundu suggests that Forbes et al. did not properly account for the incipient resolution of the clusters. This may well indicate that the data were only marginally suitable for resolving the bimodality and that differences in the details of the reduction could make the difference in whether the peaks were resolved.

\subsection{Metallicity}

It is of great interest to translate the colors of the GCs into metal abundances in order to examine the chemical enrichment history of the entire GCS and its components, as well as to compare these values with those for other GCSs. The integrated broadband colors of GCs can be transformed into metallicity, under the hypothesis that the populations are old enough that age effects on the colors are much smaller than metallicity effects and that the GCs are similar to the metallicity calibrators in their abundance characteristics. For the Washington system, the first assumption is fulfilled for populations older than $\sim 5-10$ Gyr (Geisler et al. 1996). Good evidence now exists from spectroscopically derived ages that GCs in galaxies such as M87 and NGC 1399 generally satisfy both criteria, especially the former (see, e.g., Cohen, Blakeslee, \& Ryzhov 1998, Kissler-Patig et al. 1998a).

Previous $V-I$ studies of the NGC 1427 GCS have derived very similar values for the mean color of the entire GCS but have used several different metallicity calibrations to transform mean color to metallicity, thus yielding a significant variation in mean GCS metallicity. Forbes et al. (1996) found a mean color of $1.04 \pm 0.01$ and derived a mean $[\mathrm{Fe} / \mathrm{H}]$ of $-0.83 \pm 0.04$. The Kissler-Patig et al. (1997) values were $1.03 \pm 0.03$ and $-0.7 \pm 0.3$, while Kundu (1999) derived $1.01 \pm 0.01$ and $-1.12 \pm 0.05$. Only in the last study was bimodality found likely, with peaks at $V-I \sim 0.9$ and 1.1, yielding metallicities (on Kundu's scale) of -1.64 and -0.70 .

An initial $C-T_{1}$ integrated color-versus-metallicity calibration was obtained by Geisler \& Forte (1990) using some 50 Milky Way clusters. This led to a linear relationship; however, the number of clusters available was limited, as was their color and metallicity range. A new $C-T_{1}$ metallicity calibration has recently been derived (Geisler \& Forte 2001) that includes a large sample of clusters associated with NGC 4486 having both spectroscopic abundances (Cohen et al. 1998) and $C-T_{1}$ colors (Lee \& Geisler 2001). This new calibration includes not only about 4 times as many GCs with good metallicities but also wider color and metallicity ranges than were available to Geisler \& Forte (1990).

The mean $C-T_{1}$ color of our background-corrected sample is 1.44 . Using the new metallicity calibration, this gives an overall metallicity of $[\mathrm{Fe} / \mathrm{H}]=-0.8$, with a total uncertainty of $\sim 0.2$ dex including photometric errors, aperture corrections, and photometric and metallicity calibration uncertainties. This value is in the middle of the range derived from the $V-I$ studies. Adopting a mean color of $C-T_{1}=1.35$ (derived from both the Gaussian analysis and the KMM test) as a representative value for the BGCs, the new calibration yields $[\mathrm{Fe} / \mathrm{H}]=-1.0 \pm 0.2$. This abundance is higher than that of the halo Milky Way clusters having a $C-T_{1}$ color peak between 1.15 and 1.20 and a mean metallicity of $\sim-1.6$ on the Armandroff \& Zinn (1988) scale, and it is also higher than that obtained by Kundu (1999) for the same NGC 1427 BGC peak. From these results it is clear that any comparative study of GCSs 
in different galaxies will first require a firmly based and consistent color-metallicity relation.

For the RGCs, the Gaussian analysis and the KMM test yield mean $C-T_{1}$ colors that differ by $0.06 \mathrm{mag}$, and we adopt their average value, $C-T_{1}=1.76$. This leads to $[\mathrm{Fe} / \mathrm{H}]=-0.15$, making the RGCs comparable in metallicity to the inner stellar population of the NGC 1427 halo (assuming that the same color-metallicity relation holds for the halo light) and slightly more metal-rich than GCs in the Milky Way bulge (Minniti 1995).

\subsection{Implications}

The combination of our results and those of Kundu (1999), who finds likely bimodality over a large range of galaxy luminosity, suggests that bimodality among LLEs may also be very common, perhaps as common as for GEs. Although bimodality may be a common feature, it should be noted that, in the case of NGC 1427, the spatial distribution of the RGCs seems much more concentrated than that of their counterparts in GEs, rather suggesting a similarity with the high-metallicity clusters observed in the bulges of disk galaxies.

As noted by Kundu (1999), the existence of bimodality in LLEs raises some problems for the Côté et al. (1998) model of galaxy formation. These authors start with elliptical galaxies with a single RGC population whose mean metallicity follows the galaxy luminosity-GC metallicity relation given in Forbes et al. (1997). The BGCs are then built up from a host of mergers or tidal stripping during encounters with smaller galaxies whose GCSs are also initially unimodal. However, our results suggest that bimodality is present in these smaller galaxies as well. In addition, the existence of a galactocentric color gradient in this population shows that the accretion process was probably not entirely without dissipation.

The NGC 1427 RGCs have a mean color and metallicity similar, within the observational uncertainties, to those of the inner galaxy halo. This similarity strongly suggests a genetic connection between these systems, as discussed by Forbes et al. (1998) and by Forbes \& Forte (2001). As these GCs are bluer than their counterparts in NGC 1399 by 0.10-0.15 mag in $C-T_{1}$ at a given radius, their chemical enrichment does seem to be smaller than that in the more massive NGC 1399.

Remarkably, the BGCs in both NGC 1399 and NGC 1427 share not only similar colors but also identical galactocentric gradients. These gradients, in turn, are also comparable to the galaxy halo gradients (although the halos are redder), suggesting that, in some way, these subsystems in each galaxy are linked in a way that still needs to be clarified.

The existence of a color gradient in the BGCs suggests that they may have been formed at an early stage involving some degree of dissipative collapse, if the color gradient is understood as a result of a metallicity variation. Alternatively, the BGCs' color gradient might be interpreted as a difference in age, with the GCs located in the inner regions being older than those in the galaxy's periphery. The color difference between the innermost and outermost BGCs, about $0.05 \mathrm{mag}$ in $C-T_{1}$, or $0.025 \mathrm{mag}$ transformed into $(V-I)_{0}$, would imply that the innermost BGCs are some 2.3 Gyr older, according to the population synthesis models by Worthey (1994). This age difference increases to $4 \mathrm{Gyr}$ if the simple stellar population models given by Buzzoni (1989) are adopted instead.

If in fact the RGCs have higher metallicity than the BGCs, those clusters likely formed after BGCs, in a metallicity environment comparable to that of the galaxy's stellar halo and as a result of either a merger or an in situ process. This picture implies younger ages for the RGCs. No evidence seems to support this scenario in the case of the M87 GCS (Cohen et al. 1998), although the effect of the limiting magnitude of their GC sample on that result remains to be evaluated. On the contrary, arguments for age differences among Milky Way clusters with different chemical abundances have been given by Rosenberg et al. (1999), suggesting that higher metallicity clusters are younger by some $17 \%$ than their lower metallicity counterparts.

We conclude that the study of the GCSs of LLEs, and of NGC 1427's GCS in particular, yields a number of clues vital to galaxy formation models. Similarly detailed investigations of other such galaxies are clearly needed to complement the studies available for their brighter counterparts. It would also be of great interest to examine the kinematics and mean ages of the RGCs and BGCs in NGC 1427 in order to help determine the cause(s) of their distinct origins.

The authors would like to acknowledge M. Kissler-Patig for suggesting that NGC 1427 would be an interesting target and for providing us with the BOOTSTAT statistical package. We would like to thank M. Navarette of CTIO for his excellent execution of our night of service observing. E. Ajhar kindly provided us with the VISTA software and instruction on its use for removing the galaxy background light. M. E. Geisler provided encouragement and constant support. D. G. acknowledges financial support for this project from CONICYT through Fondecyt grants 1000319 , 7000319 , and 8000002 and from the Universidad de Concepción through research grant 99.011.025. Support for this work was also provided to A. E. P. by the NSF through grant GF-1003-98 from the Association of Universities for Research in Astronomy, Inc., under NSF cooperative agreement AST 89-47990, and to J. C. F. by the Agencia Nacional de Promoción Científica y Tecnológica (Argentina).
Armandroff, T. E., \& Zinn, R. 1988, AJ, 96, 92

Ashman, K. M., Conti, A., \& Zepf, S. E. 1995, AJ, 110, 1164

Ashman, K. M., \& Zepf, S. E. 1992, ApJ, 384, 50

Barmby, P., Huchra, J. P., Brodie, J. P., Forbes, D. A., Schroder, L. L., \& Grillmair, C. J. 2000, AJ, 119, 727

Buzzoni, A. 1989, ApJS, 71, 817

Canterna, R. 1976, AJ, 81, 228

Cohen, J. G., Blakeslee, J. P. \& R yzhov, A. 1998, ApJ, 496, 808

Côté, P., Marzke, R. O., \& West, M. J. 1998, ApJ, 501, 554

Davies, R. L., Sadler, E. M., \& Peletier, R. F. 1993, MNRAS, 262, 650

Forbes, D. A., Brodie, J. P., \& Grillmair, C. J. 1997, AJ, 113, 1652

Forbes, D. A., \& Forte, J. C. 2001, MNRAS, in press

\section{REFERENCES}

Forbes, D. A., Franx, M., Illingworth, G. D., \& Carollo, C. M. 1996, ApJ, 467,126

Forbes, D. A., Grillmair, C. J., Williger, G. M., Elson, R. A. W., \& Brodie, J. P. 1998, MNRAS, 293, 325

Forte, J. C., Pellizza, L., \& Faifer, F. 2001, in preparation

Forte, J. C., Strom, S. E., \& Strom, K. 1981, ApJ, 245, L9

Gebhardt, K., \& Kissler-Patig, M. 1999, AJ, 118, 1526

Geisler, D. 1996, AJ, 111, 480

Geisler, D., \& Forte, J. C. 1990, ApJ, 350, L5 2001 , in preparation

Geisler, D., Lee, M. G., \& Kim, E. 1996, AJ, 111, 1529

Harris, W. E., \& van den Bergh, S. 1981, AJ, 86, 1627 
Harris, W. E., Harris, G. L. H., \& McLaughlin, D. E. 1998, AJ, 115, 1801

Kissler-Patig, M., Brodie, J. P., Schroder, L. L., Forbes, D. A., Grillmair, C. J., \& Huchra, J. A. 1998a, A.J, 115, 105

Kissler-Patig, M., Forbes, D. A.. \& Minniti, D. 1998b, MNRAS, 298, 1123

Kissler-Patig M., \& Gebhardt, K. 1998, AJ, 116, 2237

Kissler-Patig, M., Kohle, S., Hilker, M., Richtler, T., Infante, L., \& Quintana, H. 1997, A\&\&A, 319, 470

Kundu, A. 1999, Ph. D. thesis, Univ. Maryland

Lee, M. G., \& Geisler, D. 2001, in preparation

Lee, M. G., Kim, E., \& Geisler, D. 1998, AJ, 115, 947

Madore, B. F., et al. 1999, ApJ, 515, 29

McLaughlin, D. E., Harris, W. E., \& Hanes, D. A. 1994, ApJ, 422, 486

Minniti, D. 1995, AJ, 109, 1663

Ostrov, P. G., Forte, J. C., \& Geisler, D. 1998, AJ, 116, 2854 (OFG)
Ostrov, P. G., Geisler, D., \& Forte, J. C. 1993, AJ, 105, 1762 (OGF)

Prosser, C. F., et al. 1999, ApJ, 525, 80

Richtler, T. 1995, Rev. Mod. Astron., 8, 163

Rosenberg, A., Saviane, I., Piotto, G., \& Aparicio, A. 1999, AJ, 118, 2306

Schweizer, F. 1986, Science, 231, 227

Searle, L., \& Zinn, R. 1978, ApJ, 225, 357

Stetson, P. B. 1987, PASP, 99, 191

. 1991, in ESO/ST-ECF Data Analysis Workshop, ed. P. J. Grosbøl \& R. H. Warmels (ESO Conf. and Workshop Proc. 38; Garching: ESO), 187

Whitmore, B. C., Sparks, W. B., Lucas, R. A., Macchetto, F. D., \& Biretta, J. A. 1995, ApJ, 454, L73

Worthey, G. 1994, ApJS, 95, 107

Zepf, S. E., \& Ashman, K. M. 1993, MNRAS, 264, 611 